\title{
LA UNIVERSIDAD DE CHILE Y SU PROYECTO FUNDADOR
}

\author{
por DARÍO OSES \\ Coordinador \\ Archivo Central Andrés Bello
}

\begin{abstract}
RESUMEN
La creación de la Universidad de Chile, en 1842, implicó el tránsito desde la tradición escolástica a la Universidad moderna. Esta transición fue una acción deliberada del Estado chileno por modernizar la sociedad, a través de la difusión del conocimiento, de sus aplicaciones y de la incorporación de los métodos que aseguraran la continuidad en la generación del mismo.
\end{abstract}

La creación de la Universidad de Chile se inscribe en el llamado "Movimiento de 1842", que fue claramente fundador de una cultura nacional, republicana y moderna. Dentro de la periodificación tradicional de la historia de Chile, este movimiento ocurre en la República Conservadora, que fue extraordinariamentefecunda, no sólo en la formación de instituciones políticas, juríáicas, educacionales, artísticas y científicas, sino también en otras creaciones más "intangibles" Así, por ejemplo, a partir de 1842 se funda una literatura auténticamente nacional, como lo indicaJosé Promis ${ }^{1}$, y también se inicia una historiografía científica, hecha sobre la base de las fuentes y del estudio crítico de ellas.

${ }^{1}$ Promis, José, Testimoniasy documentas de la literatura chilena(1842-1975), Editorial Nascimento, Santiago, 1977,pp. 30-31.

A este respecto, el autor anota "Solamentese puede hablar de una literaturanacional en la medida en que se toma conciencia de su realidad o, como ocurrió en 1842, cuando se toma conciencia de la necesidad de crearla [...] Los escritores de la generación de Lastarria comenzaron su tarea intelectual con la muy clara noción de ser los primeros y de la responsabilidad que asumían al convertirseen los fundadores. Si bien es cierto no fueron los primeros en escribir, lo hicieron reflexionando sobre lo que esto significaba. En justicia, son los verdaderos fundadores de la tradición literaria nacional". 
La organización de la Universidad de Chile, que se convirtió en modelo de la moderna Universidad americana, fue posible gracias a la conjunción de una cantidad de factores. Entre éstos se cuenta la gravitación creciente que la filosofía de la Ilustración había venido alcanzado en el país desde la época de Carlos III. Como se sabe, desde sus inicios el reformismo ilustrado - qu e se impulsa hacia América desde la España del siglo xviI-, proclama su entusiasmo por el desarrollo de las modernas disciplinas cientificas; por su aplicación al progreso material, a la transformación racional de las sociedades humanas, y también a la perfectibilidad moral del hombre; $\mathbf{y}$, por último, por el papel que correspondía al Estado como agente activo en la difusión del conocimiento, principalmente a través de la enseñanza.

Otro factor importante fue la estabilidad poítica de la nación chilena, que, al coincidir con un período de anarquía en casi todo el resto del continente americano, hizo posible la llegada a nuestro territorio de algunas de las más brillantes figuras intelectuales americanas de la época, como Domingo Faustino Sarmiento, Bartolomé Mitre, Vicente Fidel López, Juan Bautista Alberdi, Juan García del Río, y sobre todo, Andrés Bello. Este grupo, junto al de la joven elite intelectual chilena, tenían conciencia de que, concluido el proceso de emancipación política, se hacía necesario dar autonomía económica, social y cultural a los países americanos e insertarlos en el concierto de las naciones civilizadas. Aun cuando no todos coincidían en los métodos para lograr este objetivo, la inmensa mayoría de ellos atribuía un valor instrumental de primera importancia a la educación ${ }^{2}$.

De esta forma Chile, o mejor dicho Santiago,se transformó en la sede del pensamiento americano.

Al fundarse la Universidad de Chile, ésta se imbrica dentro de la tradición mundial de la Universidad. Hacia mediados del siglo xTx, estas Corporaciones habían recuperado su categoría de principales instituciones de la cultura en el mundo occidental, luego de una crisis que estuvo a punto de eliminarlas de la escena cultural y social.

La Universidad aparece en el siglo xI como producto de un largo proceso que se inicia con el colapso del mundo antiguo. Desde entonces,

${ }^{2}$ Tal vez la Única voz disidente fue la deJuan Bautista Alberdi, para quien las escuelas primarias, los liceos y Universidades no eran por sí solos, y sin las grandes empresas productivas, instrumentos muy eficaces de progreso. Este argumento sería llevada a sus términos má s extremos por distintos sectores que, en diversas épocas, vienen repitiendoque las empresasproductivas por sí solas son los agentes del progreso,y que pueden prescindir completamentede las Universidades. 
en monasterios e iglesias se desarrolla un esfuerzo vasto, paciente y anónimo, por rescatar los valores culturales de la Antigüedad Clásica. La sistematización de esta tarea va conduciendo a la formación de las primeras universidades: Bolonia y Salerno, dedicadas al cultivo y enseñanza de una sola disciplina - el Derecho y la Medicina, respectivamente - y luego París, que se organiza sobre la base de Facultades que pretenden comprender, en conjunto, todo el universo del conocimiento.

La tradición universitaria europea llega al país, en primer término, a través de las Universidades conventuaies que se fundaron en Chile en las primeras décadas del siglo xvi. Luego por la Universidad de San Felipe, que fue una Universidad bajo patronazgo real, docente y de claustro, y quedó habilitada para impartir los mismos cursos que se daban en Lima, México o Sdlamanca. Por Último, las influencias de las modernas Universidades europeas llegaron a través de algunos criollos americanos que vivieron en Europa en las primeras décadas del siglo xIx, y que se preocuparon de las instituciones culturales del viejo mundo. Entre éstos destaca Andrés Bello, quien tuvo oportunidad de conocer los modelos universitarios de Inglaterra, Escocia, Alemania y Francia, y que después tendría una influencia decisiva en la cultura y en la vida pública nacionales. De esta forma, la Universidad criolla va siguiendo el desarrollode los paradigmas europeos, e incluso tiende a reproducir, con cierto retraso, sus ciclos de decadencia y renovación. Con la creación de la Universidad de Chile, sin embargo, se hace un intento seriopor crear una Universidad adecuada a las necesidades de los países americanos que estaban iniciando su vida independiente, y que no tenían grandes recursos humanos ni materiales que dedicar a la enseñanza y la cultura.

En el conocido discurso de instalación de la Universidad de Chile, Andrés Bello se refirió al estatuto orgánico de 1842 como a la ley que había restablecido "la antigua Universidad sobre nuevas bases, acomodadas al estado presente de la civilización y a las necesidades de Chile".

La Universidad de Chile, en efecto, se refunda en 1842, y entre ella y la antigua Real Universidad de San Felipe existe al menos una continuidad jurídica. El cambio de estatuto orgánico implica, sin embargo, una transformación radical, desde una Universidad como la de San Felipe que, a pesar de la modernización de los estudios que se intentó en la época del Despotismo Ilustrado, seguía arrastrando el peso de las tradiciones escolásticas, a una Universidad moderna.

Al aludir al "estado presente de la civilización",Bello se refería a la realidad que se vivía en el país y en el mundo, en los momentos en que se organiza la Universidad de Chile. Entonces en el orbe occidental se 
desarrolla, por una parte, la consolidación de los Estados Nacionales y, por otra, la llamada Revolución Industrial. Por lo tanto, cobran creciente importancia los progresos de la ciencia y sus aplicaciones, y la formación de profesionales calificados para desempeñarse en las labores productivas, en la judicatura y la administración del Estado.

$\mathrm{Al}$ dar las primeras noticias acerca del carácter que tendría la Corporación, en un artículo publicado en el diario "El Araucano", Andrés Bello indicaba claramente que esta Universidad no iba a ser un establecimiento escolástico, dedicado a las cienciasespeculativas, que no pueden aplicarse al progreso de la sociedad actual.

Al recalcar que el nuevo establecimiento no se dedicaría a las ciencias cimentadas en la autoridad del dogma, Bello estaba situándolo dentro del proceso de transformación que vivió la Universidad en Occidente, y que se inicia cuando los humanistas del Renacimiento se plantearon críticamente frente al excesivo formalismo de la tradición escolástica. A pesar de estas críticas, las Corporaciones Universitarias se habían mantenido por mucho tiempo cerradas a la nueva visión del mundo, construida sobre la observación, la experimentación y cuantifkación. En efecto, las Universidades tradicionales europeas se mostraron incapaces de responder a las riquísimas provocaciones derivadas del desarrollode las modernas ciencias exactas y naturales, que conformaban una nueva forma de ver y de conocer el mundo, y, además, de actuar sobre él.

Las Universidades españolas, modelo de las americanas durante el período indiano, no fueron una excepción a este estado de atraso y estancamiento. El profesor Alamiro de Ávila indica que a partir de la segunda mitad del siglo xvII, la independencia y privilegios que habían alcanzado las Universidades peninsulares y sus colegios mayores les impedian impulsar mejoras. La Facultad básica, que era la de Artes o Filosofía, cuyos grados eran requisito para seguir estudios en las Facultades de Teología, Leyes, Medicina y Matemáticas, estaba estancada en un aristotelismo de segunda mano - ya que ni siquiera se discutían en ella las obras de Aristóteles sino las de sus comentadores - , y aprisionadapor el formalismo silogístico ${ }^{3}$.

La Facultad de Teología seguía el mismo método de razonamiento silogístico. En la de Derecho se estudiaba el Derecho Romano y Justineaneo, y el Derecho Canónicono vigente, sin tocar el Derecho Nacional que

3 Ávila Martel, Alamiro de, "LaUniversidady los estudios superioresen Chile en la época de Carlos III", en Campos Hamet, Pernando \& al., Estudiossobre la época de Carlos III en el Reino de Chile, Ediciones de la Universidad de Chile, Santiago, 1989,pp. 173-174. 
era el que los graduados usarían en el ejerciciode su profesión. Finalmente, la enseñanza de la Medicina y de las Matemáticas se hacía sobre la base de la autoridad del dogma, con total ausencia de experimentación y conocimiento de las investigaciones més recientes en estas disciplinas.

Las nuevas tendencias científicas, especialmente en ciencias naturales y exactas, se desarrollaron por mucho tiempo fuera de las Universidades, en las academias y sociedades científicas, que comienzan a fundarse durante el siglo XVII, primero en Inglaterra y Francia, y luego en el resto de Europa. Así, por ejemplo, en España, Felipe V funda las primeras Academias Reales. Las carencias o la ausencia total de la enseñanza del Derecho vigente en las Universidades, se suplen en las Academias de Leyes y Práctica Forense.

En Europa y América las Universidades tendieron a parapetarse en la defensa de sus privilegios y de sus anquilosadas tradiciones, resistiendo la apertura a las nuevas ciencias y métodos de conocimiento, y a los requerimientos de sociedades cuyo progreso necesitaba de conocimientos útiles ${ }^{4}$.

Ia Revolución Francesa, al instaurar una sociedad moderna e igualitaria, redujo drásticamente esos privilegios y terminó con la Universidad tradicional. Como apunta el profesor Ricardo Krebs, "Napoleón asignó a la Universidad francesa la tarea de formar a los profesionales que necesitaba para la administración civil, judicial y militar en su imperio"5. Paralelamente con esta Universidad orientada prioritariamente a la formación profesional, aparece la Universidad científica de Berlín, que es creación de Guillermo de Humboldt.

Una de las características de la ciencia moderna es su carácter acumulativo, que le confiere una aceleración que se va acrecentando en la medida en que existe mayor conocimientodisponible. Las ciencias naturales y exactas se construyen sobre sí mismas, sobre sus propios resultados previos. Pero, al mismo tiempo, estos resultados son siempre provisorios.

${ }^{4} \mathrm{La}$ discusión sobre si la Universidad debe estar en la feria, es decir expuesta al mundo y a sus exigencias, o si, por el contrario, debe encerrarseen su propia torre de marfil, se ha repetido más de una vez a lo largo de la historia. Sería importante examinar críticarnente esta tendencia de la Universidad a "enclaustrarse"es decir, a cerrarse sobre sí misma, loque ocasiona crisis de legitimidad, o sentimientos de culpabilidad que algunas veces se han resuelto en reacciones hacia el extremo opuesto, es decir, hacia la apertura total. Así, por ejemplo, las Universidades latinoamericanasde los "60 y 70 de este siglo, vivieron la obsesión por la permeabilldad hacia lo que llamaban "elmedio social".

5Krebs, Ricardo, "Ideay desarrollo de la Universidad en Occidente", en Cuademos Consejo de Rectores Universidades Chilenas, N 156, enero-abril de 1982, Santiagode Chile, p. 15. 
La Universidad de Berlín aceptó desde el principio este carácter dinámico de la ciencia. Por ello puso el énfasis de su formación no en la transmisión de conocimientos, sino en desarrollar la capacidad de someter a revisión los resultados obtenidos y de generar conocimientos nuevos; en síntesis, a formar una mentalidad esencialniente abierta, crítica, receptiva, creadora.

Como lo señala el profesor Ricardo Krebs, en el modelo de la Universidad de Humboldt, "elprofesoruniversitario debía enseñar lo que estaba investigando y debía hacer participar al estudiante en el proceso creador de la elaboración de nuevos conocimientos. La enseñanza universitaria, más que transmisión de resultados, debía ser la iniciación en el quehacer científico"6.

La Universidad de Chile nace o renace en 1842 con un espíritu de apertura a las transformaciones que inevitablemente genera en la saciedad el desarrollo de la ciencia moderna. La Universidad era vista como uno de los agentes a través de los cuales debería transferirse hacia la sociedad este dinamismo inherente a la ciencia, que se percibía como diametralmente opuesto al carácter estacionario de las disciplinas basadas en la tradición escolástica, a las que se asociaba con una sociedad ordenada de acuerdo con jerarquías inmóviles.

Es ciertoque en el país, durante la época de Carlos 111, se habían hecho intentos serios por modernizar la enseñanza superior y darles aplicación práctica a los contenidos de los estudios. Estos intentos se realizaron principalmente en los colegios universitarios, como el Convictorio Carolino, la Academia de Leyes Reales y Práctica Forense, y sobre todo en la Academia de San Luis, regida por una de las mayores figuras criollas del Despotismo Ilustrado: don Manuel de Salas.

El profesor Alamiro de Ávila da noticias sobre el curso de Física de Manuel Antonio Talavera, en el ConvictorioCarolino, en el que se describen y comentan experimentos y autores como Newton, Boyle, Wolf, Gassendi, Descartes, Galileo y Kepler. Asimismo, se refiere a la obra del dominico Sebastián Díaz, Noticia general de las cosas del mundopor el orden de su colocación. Para el uso de la casa de los señores Marqueses de Pica y para instrucción común de lajuventud del Reino de Chile, impreso en Lima, en 1783. El padre Díaz fue miembro del claustro de la Universidad de San Felipe y examinador en Teología. En su obra, el dominico critica la forma escolástica y expone los contenidos de las ciencias con un pensamiento moderno, comentando, nuevamente, las 
ideas de Newton y, entre otras materias, la evolución de la Cosmografía, desde Tolomeo a Kepler?.

Por su parte, el historiador Mario Góngora revisó las ideas modernizadoras que en materia de educación y cultura tuvieron algunas figuras descollantes del Despotismo Ilustrado en Chile, como Miguel José Lastarria, Juan Martínez de Rozas, Mateo Zambrano, Juan Egaña,José Antonio Errázuriz y Manuel de Salas ${ }^{8}$.

Aun así, la Universidad de San Felipe siguió asociada a la idea de la tradición escolástica y del "Antiguo Régimen", y esto explica la-desconfianza con que se la miró en la época republicana, la decadencia que sufrió durante este períodoy, finalmente, la total transformación a que se la sometió con la ley orgánica de 1842.

Andrés Bello dio a la Universidad de Chile su fisonomía moderna y su doctrina singular. Más de una vez se ha dicho, con cierta ligereza, que la Universidad de Chile fue una copia de elementos tomados de las Universidades inglesas y escocesas, de la Universidad de Humboldt y de la napoleónica. Desde luego no es una invención absolutamente original, porque no podría haberse creado una Universidad desgajada de la tradición cultural de Occidente. Pero los aportes de los modelos universitarios europeos están adaptados a las necesidades de los pueblos y a la realidad de las novísimas Repúblicas americanas. Así, por ejemplo, Bello se hace cargo de la orfandad científica en que se encuentran estos pueblos y se plantea problemas que han permanecido vigentes hasta ahora, como si debemos recibir sin crítica la ciencia de los países europeos, o, por el contrario, aprender a pensar.

Las primeras noticias acerca del carácter de la nueva Corporación las entrega Bello en "El Araucano", en 1842, cuando el proyecto de la Ley Orgánica de la Universidad fue aprobado por el Consejo de Estado y remitido al Poder Legislativo.

"No se trata de aquellos establecimientos escolásticos o de ciencias especulativas, destinados principalmente a fomentar la vanidad de los que deseaban un título aparente de suficiencia, sin ventajas reales e inmediatas para la sociedad actual [...1 Se desea satisfacer,en primer lugar, una de las necesidades que más se han hecho sentir desde que con nuestra emancipación política pudimos abrir la puerta a los conocimientos útiles, echando las bases de un plan general que abrace estos conoci-

${ }^{7}$ Ávila Martel, Alamiro de, op. cit.

${ }^{8}$ Góngora del Campo, Mario, 'Notaspara la historia de la educación universitaria colonial en Chile",en Anuario de Estudios Americanas, tomo v, Sevilla, 1949. 
mientos, en cuanto alcancen nuestras circunstancias, para propagarlos con fruto en todo el país y conservar y adelantar su enseñanza de un modo fijoy sistematizado, que permita, sin embargo, la adopción progresiva de los nuevos métodos y de los sucesivos adelantos que hagan las ciencias" $\%$.

De esta forma, Bello le entregaba a la nueva Universidad una misión que es propia del ideario de la Ilustración: el cultivo, enseñanza y propagación, de los llamados "conocimientosútiles". Habla de la "adopción progresiva de los nuevos métodos", es decir, del método científico que desplazaría al escolástico. Esta adopción aparece como condición necesaria para seguir incorporando "los sucesivos adelantos que hagan las ciencias".Todo esto con el fin de obtener "ventajasreales", es decir, progreso para la sociedad.

"Se echaba de menos, en segundo lugar, un cuerpo conservador, por decirlo así, de estos conocimientos que, bebiendo en las fuentes y manteniendo las buenas doctrinas, alejase de entre nosotros el empirismo, sin permitir que el mediano saber o el superficialismo, tal vez más fatales para las naciones que la ignorancia, ocupen el lugar del verdadero mérito, que sólo puede ser puesto a la prueba y generalmente reconocido por medio de estos cuerpos científicos.

"Senecesitaba igualmente de una academia en que pudiesen cultivarse y brillar los ingenios nacionales, que frecuentemente nacen entre nosotros para morir pronto por falta de un teatro aparente y digno y por falta de aquellos estímulos de honor y gloria que sólo pueden alentar a las almas elevadas y generosas, y que son en todas partes la vida de las ciencias y de las artes liberales. Estas mismas ciencias y artes apenas tienen aplicación entre nosotros; $y$ ninguna carrera segura aparece abierta al joven estudioso que las ha cultivado con esmero y aprovechamiento,y que se ve obligado, por lo mismo, a interrumpir el curso de sus penosas tareas para dedicarse a ctra vocación ajena. Al cuerpo universitario está reservado presentar un vasto campo de acción en la carrera de los conocimientoshumanos, estimular su cultivoy coronar su mérito: él debe poner en honor las ciencias y demostrar a los hombres industriosos en particular, como a la comunidad entera, que sin ellas no hay verdaderos y sólidos adelantos, que ellas son el manantial de todas las riquezas"10.

9Bello, Andrés, 'Establecimientode la Universidadde Chile", en Obras completas de Andrés Bello, edición hecha bajo la dirección del Consejo de Instrucción Pública, vol. viI, "Opúsculos literarios y críticos III", Santiago de Chile, 1885,p. 278.

${ }^{10}$ Bello, Andrés, op. cit., p. 279. 
En estos párrafos $\boldsymbol{s}$ encuentra una de las claves del proyecto modernizador que emprendería la Universidad en la sociedad chilena. Al dar las oportunidades para que se manifiesten las mejores capacidades humanas, al "poner en honor las ciencias", al estimular el cultivo de los conocimientosy "coronarsu mérito", se estaban creando las bases de una nueva forma de movilidad social. Ésta, posteriormente, con el desarrollo de las profesiones, permitiría la consolidación de una clase media que iba a cumplir con la democratización del país, deliberadamente postergada por los regimenes presidenciales autoritarios y fuertes que se prolongaron prácticamente hasta 1891.

En su conocido discurso, pronunciado en la ceremonia de instalación de la universidad, en septiembre de 1843, Bello trazó lo que él mismo llama "programaenteramente chileno" para la Universidad de Chile. En este programa indica claramente las necesidades a las que debería responder la Corporación.El énfasis sigue poniéndose en los "conocimientos útiles": "La utilidad práctica, los resultados positivos, las mejoras sociales, es lo que principalmente espera de la Universidad el Gobierno"11.

Dentro de este programa, incluso los estudios de la Facultad de Teología estaban orientados a formar "dignosmiembros del culto"y a proveer "de competente educación religiosa y moral". "La moral (que yo no separo de la religión) es la vida misma de la sociedad", anotaba Bello ${ }^{12}$.

"La Universidad —dice más adelante — examinará los resultados de la estadística chilena, contribuirá a formarla y leerá en sus guarismos la expresión de nuestros intereses materiales. Porque en éste como en los otros ramos, el programa de la Universidad es enteramente chileno: si toma prestadas a la Europa las deducciones de la ciencia es para aplicarlas a Chile. Todas las sendas en que se propone dirigir las investigacionesde sus miembros, el estudio de sus alumnos, convergen a un centro: la patria"13.

\footnotetext{
${ }^{11}$ Bello, Andrés, Discurso de Instalación de la Universidad de Cbile, Oficina de Educación Iberoamericana, Madrid, 1981, pág. 28.

${ }^{12}$ Recordemos que uno de los intentos del reformismo ilustrado español fue el de conciliar las nuevas tendencias de la ciencia y de la iluminación racionalista con la religión. Esta aspiración también se halla presenteen Bello, que era un católicoobservante.Es así comoen el discurso de 1843 afirmaba: "Calumnianno sé si diga a la religióno a las letras, los que se imaginan que puede haber una antipatía secreta entre aquéllasy éstas. Yo creo, por el contrario,que existe, que no puede menos que existir, una alianza estrecha entre la revelación positiva y esa otra revelación que habla a todos los hombres en el libro de la naturaleza".
}

${ }^{13}$ Bello, Andrés, op. cit.,p. 29. 
En otra parte de su discurso, al detallar los estudios a que habrá de dedicarse cada Facultad, indica: 'La Medicina investigará [...] las modificaciones peculiares que dan al hombre chileno su clima, suscostumbres, sus alimentos; dictará las reglas de la higiene pública y privada; se desvelará por arrancar a las epidemias el secreto de su germinación y de su actividad devastadora"14. A continuación alude a "lasutilidades positivas de las Ciencias Matemáticas y Física" y a "sus aplicaciones a una industria naciente".

Advirtió, sí, Bello, que al fomentar las aplicaciones prácticas estaba lejos de menospreciarel valor de "conocimientode la naturaleza en todos sus variados departamentos". Esto, en primer lugar, porque "para guiar acertadamente la práctica, es necesario que el entendimiento se eleve a los puntos culminantesde la ciencia"15.

En la memoria que leyó con ocasión del aniversario de la Universidad, el 29 de octubre de 1848, después de un largo recuento de los progresos que se habían logrado en la educación pública, así como de los problemas que había encontrado la Universidad en su función de Superintendencia de Educación, Bello señalaba que "nose debe olvidar que nuestra ley orgánica [...] ha encargado a la Universidad no sólo la enseñanza, sino el cultivo de la literaturay las ciencias; ha querido que fuese a un tiempo Universidad y Academia; que contribuyese por su parte al aumento y desarrollo de los conocimientoscientíficos; que no fuese un instrumento pasivo, destinado exclusivamente a la transmisión de los conocimientos adquiridos en las naciones más adelantadas, sinoque trabajase, como los institutos literarios de otros pueblos civilizados, en aumentar el caudal común"16.

Proponerse contribuir a este "caudalcomún" del conocimientoera una audacia en aquella época para un país latinoamericanoque sólo hacía poco se había organizado como República. Costó mucho tiempo y energías terminar con la resistencia hacia la actividad científica, que incluso se sigue considerando hoy por algunos sectores como un lujo fuera del alcance de una nación en desarrollo. Por eso Bello pregunta: "¿Hay algo de presuntuoso, de inoportuno, de superior a nuestras fuerzas como han supuesto algunos? ¿Estaremos condenados todavía a repetir servilmente

${ }^{14}$ Bello, Andrés, op. cit., p. 29.

${ }^{15}$ Bello, Andrés, op. cit., p. 30.

${ }^{16}$ Bello, Andrés, "Memorialeída por el Rector de la Universidad de Chile en el aniversariosolemne de 29 de octubre de 1848", en Anales de la Universidad de Cbile, correspondientes al año 1848, mayo de 1850,Santiago,pp. 165-166. 
las lecciones de la ciencia europea, sin atrevemos a discutirlas, a ilustrarlas con aplicacioneslocales, a darles una estampa de nacionalidad?Si así lo hiciéramos seríamos infieles al espíritu de esa misma ciencia europea y le tributaríamos un culto supersticiosoque ella misma condena"17.

Bell reconocía, a continuación, que hay ramos en que por el momento sólo debíamos limitamos a admitir los resultados de la experiencia y del estudio ajenos. Otros, en cambio, exigen investigaciones locales. Citaba como ejemplos los casos de la historiografía nacional, de la Medicina y de la Fistoria Natural que, apuntaba, " es pura observación, aun para adquirir las primeras nociones se trata de ver, no las especies de que nos habían los textos europeos, sino las especies chilenas, y el árbol que crece en nuestros bosques, la flor que se desenvuelve en nuestros valles y laderas, la disposición y distribución de los minerales en este sueloque pisamos....iNo seremos capaces nosotros de hacer en el siglo xIx lo que hizo en el xvi el jesuita español JoséAcosta, cuya Historia natural y moral de las Indias, fruto de observaciones personales, es consultada todavía por el naturalista europeo?Y si lo somos, ¿se condenará como inoportuna la existencia de un cuerpo que promueva y dirija este cultivo de las ciencias?"18.

"La ciencia europea nos pide datos-continuaba argumentando Bello¿no tendremos siquiera bastante celo y aplicación para recogerlos? ¿No harán las Repúblicas americanas en el progreso general de las ciencias más papel, no tendrán más parte en la mancomunidad de los trabajos del entendimiento humano que las tribus africanas o las islas de Oceanía?"19.

La Universidad de Bello se organiza sobre la base de cinco Facultades academias, las cuales no tenían funciones docentes, y cuyo objetivo era el desarrollo de las disciplinas científicas. La principal gratificación que ofrecían estas academias era la valoración social de la dedicación al conocimiento ya que el trabajo de los académicos se hacía en términos honoríficos, y fuera de los premios establecidospara algunos certámenes, y la eventual publicación de los trabajos de investigación en los "Anales de la Universidad de Chile", no existía ningún otro tipo de recompensa.

Como lo señala Sol Serrano, estas Facultades academias resultaron "unidadesorganizativas relativamente débiles como para ser soportes de la investigación científica"20. Por otra parte, la labor científica de la

${ }^{17}$ Bello, Andrés, op. cit., p. 165.

${ }^{18}$ Bello, Andrés, op. cit., p. 167.

${ }^{19}$ Bello, Andrés, op. cit., p. 168.

${ }^{20}$ Serrano, Sol, Universidad y nación. Cbik en el siglo xIX, Editorial Universitaria, Santiago, 1993, p. 111. 
Universidad recibió muy escaso apoyo del Congreso, que veía más utilidad en el financiamiento de la educación.

La misma autora indica que si bien Ignacio Domeyko compartía con Bello la idea de una Universidad dedicada a un objetivo científico, el sabio polaco "aspiraba a la formación de un académico profesional, inexistente en Chile"21. Opinaba Domeyko que un hombre, por muy ilustrado que fuese, si se encontraba inmerso en los negocios y en las exigencias del mundo, no podría competircon otro que, al estar dedicado a la docencia, necesariamente tendría que tratar diariamente las materias de su disciplina y seguir los procesos y progresos de la ciencia.

La actividad que tal vez contribuyó en mayor medida a legitimar la utilidad social de la Universidad de Chile en sus primeros años fue la de Superintendenciade Educación, función que quedó radicada en la Facultad de Filosofía. A través del ejercicio de esta labor, la Universidad prácticamente creó un sistema de educación, proponiendo textos, diseñando planes de estudios y métodos pedagógicos y ocupándose de la dirección y control de toda la educación pública.

El consenso que existía en cuanto a los beneficios sociales que entregaba la extensión de la enseñanza, hizo que esta labor de Superintendencia fuera percibida como la más útil que la Universidad prestaba al país. No ocurrió lo mismo con la labor científica. Ésta no mereció reparos en tanto no demandara grandes gastos, pero tampoco consiguióentusiasmar a la sociedad chilena, más allá de las reducidas elites intelectuales.

La idea de Bello de una Universidad dedicada al cultivo de las ciencias y de sus aplicaciones a los grandes problemas del desarrollo nacional, tendría aún que esperar. La verdadera institucionalización de la ciencia como parte de las funciones propias de la Universidad, y la profesionalización de la labor del científico, sólovinieron a cumplirse a mediados del siglo xx, bajo la rectoría del profesor Juan Gómez Millas.

Como lo indica Sol Serrano, en el siglo xxx la docencia más que la academia fue el núcleo de la institucionalidad científica, y el profesor "el tipo social más especializado en la estructura del sistema intelectual de ese siglo. Por esto la Universidad debía ser docente"22.

Con la creación de una sección universitaria en el Instituto Nacional, hecha a propuesta de Domeyko y aprobada por el Consejo Universitario en 1847, la Universidad inició un lento tránsito que finalmente llevó a la promulgación de un nuevo estatuto orgánico, el de 1879, el que le

${ }^{21}$ Serrano, Sol, op. cit., p. 107.

22Serrano, sol, op. cit.,p. 124. 
devolvió la función docente. La Universidad de San Felipe había tenido este carácter, aun cuando permaneció ajena a la enseñanza directa de las profesiones. En efecto, en sus Facultades sólo se enseñaba desinteresadamente una ciencia o disciplina, aun cuando éstas tenían alguna relación con las principales profesiones de la época.

La docencia que comenzó a impartirse en la Sección Universitaria del Instituto Nacional y luego en la Universidad misma estaba, en cambio, directamente vinculada con la formación profesional. Esto no significó, sinembargo, la reducción de la actividad universitaria al simple esquema de fábrica de profesionales. Domeyko manifestó en su tiempo ideas que hasta hoy conservan vigencia sobre lo que debe ser la auténtica formación universitaria: 'Todaprofesión científica desciende a ser puro oficio, incapaz de marchar con el progreso del país, si se la quiere limitar a conocimientosestrictamente indispensables para su ejercicio... ${ }^{23}$. Así, el plan de organización de los estudios universitarios de 1847 se preparó, en palabras del sabio polaco, para que "seaprenda a estudiar, a pensar y racionar, de manera que en cualquiera situación de la vida en que se halle después el hombre, sabrá estudiar, recordar y proseguir el cultivo de los ramos que le sean més necesarios y útiles"24. Así, Domeyko estaba adelantando ya los modernos objetivos de aprender a aprender que se plantean actualmente para la educación.

En este punto vuelven a coincidir los propósitos de Domeyko con los del magisterio de Bello, que instaba a la juventud a aprender a juzgar por sí misma, a aspirar a la independencia de pensamiento. Esta independencia requería el respaldo de una capacidad académica para enfrentar los grandes problemas que planteaba el desarrollo integral del país, y la formación de esa capacidad fue uno de los objetivos que se mantuvo bajo los diversos regímenes orgánicos -de 1842, 1879 y 1931-que constituyeron una Universidad científica, una docente, y una de doble función, respectivamente.

Desde los tiempos de Bello, la Universidad de Chile fue la Universidad del país. A través de ella el Estado chileno impulsó su proyecto de modernización de la sociedad, por la incorporación de la cienciay de sus aplicaciones, de la generación de conocimientos, o de la formación de profesionalesy graduados. Asimismo, la fundación de la Universidad, en 1842, significa de alguna forma la consagración del prestigio de las

23Domeyko, Ignacio, "Reseñade los trabajos de la Universidad desde 1855 hasta el presente". Memoria presentada al Consejode la Universidaden sesióndel 4 de octubre de 1872.Santiago, 1872, p. 8.

${ }^{24}$ Domeyko, Ignacio, op. cit., p. 8. 
ciencias experimentales como medio fundamental de conocimiento, y el definitivo desplazamiento del método escolástico.

Más tarde, a través de la Universidad de Chile, el Estado cumplió ctras funciones, como la difusión de los valores y contenidos de la cultura y las artes hacia las grandes audiencias extrauniversitarias,o la extensión de la enseñanza superior a todo el país, como parte de programasde desarrollo regional, que pretendían otorgar oportunidades a los habitantes de las provincias.

La incorporación de nuevas funciones hizo que la Universidad creciera en tamaño y en complejidad. Esto, desde luego aumentó sus costos. Aun así, nunca se puso en duda la legitimidad de su existencia, ni siquiera cuando los fenómenos de masificación e ideologización generaron críticas que terminaron con la intervención de la Universidad por parte del Gobierno Militar. Pero este enjuiciamiento apuntaba a que se habría desvirtuado la labor universitaria y a la supuesta necesidad de aplicar los correctivos para devolver la "virtud " o " pureza" perdidas a dicha labor.

Sólo en los últimos años, cuando tiende a volatilizarse el Estado y se produce una crisis de reconocimiento social que afecta a las grandes instituciones de la República; cuando una subculturade masas se apodera de las audiencias de la cultura universitaria, y cuando con el quiebre de los grandes discursos históricos se pierde el sentido de proyecto nacional, es que la Universidad parece perder su perfil y su justificación social.

Se argumenta que todas sus funciones pueden realizarlas en mejor forma ctras instituciones. Que la docencia de pregrado pueden impartirla Universidades particulares, que entregan sólo unas pocas carreras, con una organización muy elemental y muy barata. Que más pertinente que la investigación universitaria es aquella que se realiza en las empresas, etc. Subyace a todos estos argumentos la idea de una sociedad fragmentada y algo caótica que producirá hordas de periodistas, psicólogos y abogados, y cuyos sensores para auscultar los futuros o los largos plazos se verán seriamente limitados.

Desde el programa planteado por Bello, la Universidad de Chile ha contribuido a dar sentidoy coherencia al país, y a vertebrargrandes tareas transgeneracionales. Si ahora se opta por un país desmigajado, sin coherencia, sin proyecto de futuro y en donde los sentidos se diluyan en transacciones de compra-venta, entonces, desde luego, la Universidad de Chile debería dejar de existir o limitarse a cumplir sólo aquellas actividades que puedan rendir más réditos en el corto plazo. Pero una decisión de esta naturaleza debiera tomarla y asumirla la sociedad chilena, convenientemente informada de sus consecuencias. 